\title{
THE CHEMICAL AND PHYSICAL CHANGES ATTENDANT UPON THE STERILIZATION OF MILK.
}

\author{
By Dr. Albert R. Leeds.
}

When milk is sterilized by heating in loosely plugged flasks immersed in a steam bath surface evaporation does not occur, and there is little or no pellicle formed on the surface, the sterilized milk presenting the same appearance, if the heat is not continued longer than one or two hours, as the original. Under the microscope the only visible changes effected in the boiled and sterilized milk is the coalescence of some of the fat globules into others of larger size, and the appearance of more shreds or coagula of separated caseinous matrer.

The differences in the behavior of raw, boiled and sterilized milk when treated with dilute acid were not by any means as striking as anticipated. It is frequently stated that the precipitate formed with the two latter, requires less acid for its formation, is formed more quickly, and is in softer, finer, more flocculent masses than the clot or curd of raw milk. But on treatment with equal quantities of dilute acid aaded in successive portions, and waiting for equal intervals, I have not been able to satisfy myself that much difference between the three milks, exists. This is especially true when the separated casein, which is present in raw milk, has already been partly removed with the skin formed on heating, and which itself tends to increase the size and density of the clots of acidulated raw milk. It is indeed very difficult to form a judgment when the undiluted milks are used, since the clots remain suspended in the remaining clondy liquid, and their size and appearance can readily be modified by manipulation and shaking. Better to see what takes place, each milk was diluted to twenty times its original volume with water, ten cubic centimetres of the 
milk being taken, and then a cubic centimetre of acetic acid which itself had been diluted ten times was added. At first the flocks in the raw milk formed a precipitate more dense and occupying at the bottom of the vessel a notably less volume, than that precipitating out of the sterilized milk. In the former case the supernatant liquid was also more opaque from unprecipitated white particles, but after standing 12 hours, these differences in the bulk of the precipitate had disappeared, while the supernatant liquid from the sterilized liquid had a white turbidity and that from the raw milk was perfectly clear. The boiled milk failed to precipitate at all, and an additional cubic centimetre of acid had to be added to before its casein was thrown down.

It is necessary to have recourse to more accurate methods to discover the nature of the changes effected by heating. And the more important and striking is the following. When ordinary raw milk is diluted with 20 times its volume of water, and the precipitate is filtered off, the dilute acid filtrate gives on boiling an additional precipitate. Milk which has been heated for an hour (sterilized), or boiled for half an hour, behaves altogether differently. It gives a larger amount of precipitate with dilute acid, but yields no further precipitate on boiling the filtrate. The precipitate obtained with dilute acid must certainly be different in character, therefore, when raw milk is used and when cooked milk is tested, even though the differences are difficult to recognize by the eye alone.

If we call the albuminoid matter first precipitated $\mathrm{C}$; that separated on boiling $A$, and that remaining in solution even after boiling the acid liquid, $P$, the result obtained by experiment on a sample of milk, raw, boiled for half an hour, and sterilized for one hour were as follows :

\section{Raw. Boiled. Sterilized.}

'Total Albuminoids 3.303 per cent. 3.481 per cent. 3.383 per cent.

C ("Caseine") 2.814 " " 3.129 " " $" 2.928$ " " "

A ("Albumen") 0.144 " " none " " none " "

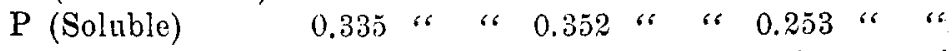

Of course the total albuminoids must have been the same ir. amount in each case, but the formation of the skin on the cooked 
milk by making it difficult to obtain a homogeneous sample, introduced slight discrepancies in the analyses.

I have put the name albumen in quotation marks, becarise while similar to the albumen of some authors, the precipitate outained in this manner would not exactly correspond. Raising the temperature merely to the boiling point is not sufficient. Some albumen is still obtained on boiling the acid filtrate from the casein-the heating must be continued for some time.

Inasmuch as gastric digestion is performed in an acid medium, and precipitation would at once occur, the more complete precipitation of the albuminoid matter in cooked as compared with raw milk would exert a corresponding influence.

The rittio of $\mathrm{C}$ to $\mathrm{A}$ and $\mathrm{P}$ appears to be very variable.

In another sample of milk obtained from the same dairy $\mathrm{C}=$ $2.213 ; \mathrm{A}=0.464 ; \mathrm{P}=0.332:$ Total $=3.519$. This gives a ratio of total albuminoids to casein of $100: \% \%$. The above sample of sterilized milk gives for the same ratio $100: 89$. It is important to note that a like change is produced in the condensation of milk for canning. Though the heating is carried on in vacuo, yet it is sufficient to raise the percentage of the albuminoids precipitable by dilute acil in the cold very notably. If the heating is continued long enough and is sufficiently raised to render the canned milk entirely sterile, the effect above noted is very evident. 'Thus in a sample of "sterilized condensed Swiss milk," the total albu" minoids were $10.599 \%$ and the casein $0.81 \%$ giving the ratio of $100: 93$.

Plate cultures of this condensed milk showed it to be entirely sterile, no bacteria colonies appearing when the plates were kept many days. And it is not surprising that this condensed milk is rendered absolutely sterile, when another evidence of prolonged heating is considered, which is the brownish color. Laboratory experiments upon the sterilization of milk in a steam bath showed that no change of color took place at the end of one hour, an hour and a half or two hours. At the end of three hours the change was perceptible and in six hours the sterilized milk had becomestrongly brownish-yellow resembling an infusion of coffee to which a lurge amount of milk has been added. Polariscopic determina- 
tions were made of the amount of milk-sugar present after each heating, the raw milk containing 4.18 per cent. No change could certainly be estimated until the end of the six hours period when the percentage had fallen to 3.94 , and it then steadily diminished until at the end of 48 hours the milk-sugar had entirely disappeared. There was a corresponding change of taste and color, the former acquiring more and more of that peculiar sweet and bitter flavor percepitable in boiled milk, and which I am inclined to attribute to the change of the milk-sugar, and the color at the same time becoming of a dark brownish-yellow. The decomposition product of the milk-sugar is the chief substance concerned in the development of the brown color, but not entirely since the mixture of casein and albumen sepurated from such heated milk and treated with ether to remove all the fat, has on drying, a strong yellow color. The casein obtained in a similar manner from raw milk dried perfectly white, and the albumen is white also. The fact that the albumen by heating becomes precipitable by dilute acid is one evidence of its change of character, and that on drying this changed albumen beomes yellow is another evidence.

The decomposition of milk-sugar is attended with the development of an acid, probably glucic acid. Filtered off from the in. soluble substances left after heating for 48 hours, the solution which no longer contained any milk-sugar, had a brown color and acidity thus developed from 100 cubic centimetres of milk was equivalent to 13.8 cubic centimetres of decinormal soda.

It may justly be objected that such great changes as the entire destruction of the milk-sugar are never met with in the condensed sterilized milk of commerce, or in commercially or privately sterilized milk. The objection while true, does not apply to the object which I had in view which was to carry the experiments far enough to find out what was the nature of the substances formed.

That the process of heating to prepare the sterilized condensed milks of commerce is not carried far enough to perceptibly lower their percentage of milk sugar may been seen from the following analyses of an American and a Swiss preparation. The American 
had a slight tint, the foreign was distinctly brownish. The condensation in both cases had been calried to such a point that the crystals of milk-sugar had begun to separate, the admixture of water bringing ont a distinctly granular appearance due, as the microscope also showed, to minute crystals of milk-sugar. I give the composition of the American variety when diluted with $2 \frac{1}{2}$ times it volume of water: when diluted to such a point that its total solids amounted to $13.4 \%$ (this being about the average total solids in our ordinary whole commercial milk); and the composition of the original condensed milk. The Swiss variety is similarly tabulated except that it was diluted for purposes of analysis with only twice its volume of water. The advertisements accompanying the samples stated that when diluter as ahovo, ench would have the strength of rich table milk. But such is not the case, and a commercial milk if prepared from them by diluting to point recommended, would be condemned by an inspector as adulterated milk.

\begin{tabular}{|c|c|c|}
\hline \multicolumn{3}{|c|}{ AUERICAN CONDENSED MILK. } \\
\hline $2 \frac{1}{2}$ times diluted. & Normal. & Original. \\
\hline Milk-sugar . . . . . 3.295 & 4.843 & $12.3 \%$ \\
\hline Fats . . . . . . . . . 2.625 & 3.859 & 9.18 \\
\hline Total Albuminoids $2.64 ;$ & 3.890 & $8 \therefore 6$ \\
\hline (Caseine...... . . 1.906 & $2.80 ?$ & ) \\
\hline Ash . . . . . . . . 0.540 & 0.802 & 1.91 \\
\hline Total Solids 9.113 & 13.394 & 31.72 \\
\hline \multicolumn{3}{|c|}{ SWISS "CONDENSED, STERILIZED CREAM MILK." } \\
\hline 2 time, diluted. & Normal. & Original. \\
\hline Milk-sugar . . . . . . 4.52'r & 4.866 & 13.581 \\
\hline Total Albuminoids 3.506 & 3.798 & 10.599 \\
\hline (Caseine $\ldots . . . . .3 .27$ & 3.515 & $9.81)$ \\
\hline Fats ............. . 3.714 & 3.992 & 11.142 \\
\hline Ash _. . . . . . . . 0.688 & 0.738 & 2.064 \\
\hline Total Solids 12.435 & 4 & 386 \\
\hline
\end{tabular}


It is surprising that the composition of two milks, as may be seen from an inspection of the column marked "Normal," should be so strikingly similar when we consider that the cattle fed on the Swiss alps, and in the Western State, from which this American sample came, were of different breed, and that their feeding, care, etc., were also widely diverse. By carrying the condensation to a farther point and by more prolonged heating, the Swiss variety was made of a slightly brown color. But both were sterile and when, thecans having been opened, diluted milk was allowed to stand in flasks loosely stoppered with cotton wool, the contents of both cans and flasks soured and spoiled very slowly.

Condensed milk properly prepared is, in fact, sterilized milk in a concentrated, convenient and portable form. It is important to compare it with sterilized milk, prepared and sold in sterilized flasks, but without condensation. One of these preparations is stated to have been prepared from the milk of registered Jersey cattle, and to be especially adapted for use by infants, children, invalids and travelers.

The advertisements state that no alterations have been in the composition of the milk, and that the only manipulation which it has undergone is that of immediate transfer to glass bottles suitably stoppered, followed by sterilization with the heat of a water bath. The temperature of the bath is not stated, nor the length of time in which the bottles are immersed, but the heat is said to be adequate tn destroy all the germs of fermentation, and that samples opened after six months have shown no change.

The advantiges claimed for this sterilized milk are its preparation from the fresh milk before any opportunity of absorption of any considerable number of microbes; that in the brief interval between the milking and the sterilizing there is no time for the development of spores; that it easily made such a close copy of nature's nourishment that only really good breast milk is its equal ; that it does not curd in solid masses, but like breast milk in small soft flakes.

In addition, the statement is made that the Jersey milk is richer in the elements needed for bone and tissue building than the milk of any other breed; that it yields an adequate and guar- 
anteed proportion of cream, averaging $25 \%$, and that when diluted with an equal volume of boiled water the "cheesy element" is reduced to a proper proportion.

Samples of this commercial sterilized milk obtained in midsummer exhibited a separation of the fats in masses of considerable size. By moderate warming and shaking this fat could be partially diffused through the milk, but not in such a manner as to bring the sterilized milk back to the ordinary appearance of rich milk, or milk on which the cream has risen on standing and then has been shaken up again with the milk. Samples which were obtained later in the year, December, did not present this appearance, but resembled rich milk, and the separation of the fat in the former case was probably due to the samples having been kept in my laboratory for several weeks during very hot weather. The reaction was neutral. Cultures were made with gelatine peptone in the ordinary manner, but no growths were obtained, showing that sufficient heat and time had been given to render the milk entirely sterile.

The composition of one of the midsummer samples was as follows:

'Total Solids determined directly ........... 14.05\%

Watel . . . . . . . . . . . . . . . . . . . . . . . . 85.95

Constituents separately determned:

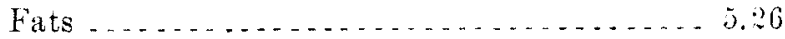

Albuminoids . . . . . . . . . . . . . . . . . 3.9\%

Milk-sugar . . . . . . . . . . . . . . . . . . . 4.20

Ash . . . . . . . . . . . . . . . . . . . . . . 0.120

Sum of constituents so deternined . . . . . . . . . . 14.166

A midwinter sample (Dec. 12th) exhibited the followng composition :

Total Solids determined directly ...........14.i0\%

Water. . . . . . . . . . . . . . . . . . . . . 85.30 
Constituents separately determined :

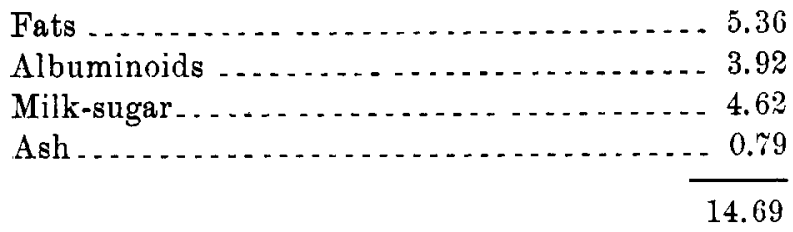

Both of these samples were sterilu. The most striking eature is their high percentage of solids, and especially of fat, as might be anticipated from the statement as to the source of the milk. Milk containing a large percentage of fat is valuable for its buttermaking qualities, but its advantages as a nutrient are open to question. Thus Uffelman (Archiv. fur die gesammte Physiologie, Vol. 29, p. 339), states, as the result of his experiments, that the milk-sugar is the constituent most completely used up in the process of digestion, the albumenoids following next, then the fat and finally the salts.

As to the apparatus used in sterilization I have employed the ordinary steam bath, which is a part of the usual Koch biological outfit, but the Arnold stenmer made and sold for milk-sterilization expressly, is more convenient and satisfactory. The flasks were closed by the rubber stoppers recommended by Soxhlet, with a gluss rod passing down through a hole in the center. But in the infant ward of the Philadelphia Hospital, the sterilized milk is heated in flasks loosely plugged with cotton wool and placed in a bath of boiling water, and this arraugement is entirely satisfactory when the milk is to be used on the spot.

As to the number and character of the bacteria present in commercial milk, it is not necessary to my present purpose to speak at great length. Five samples purchased for me at the most untidy shops, in the poorest quarters of Jersey City, yielded after 36 hours culture $33600,42000,2 \pm 7 \% 0,5832$, and 154 colonies. The smallest numbers were obtained in the last two samples which were the richer milks laving a yellowish-white color, while the other three were poor white milk.

Very interesting in this connection are the results obtained by the examination of the milk obtained from cows fed on brewers' 
grains, and a mixture of grains and swill. An analysis of the brewers' grains showed that they were rich in albuminoids, and in so far contained a large amount of material available for nutrition. Two samples were analyzed :

I.

Water .74 .81 per cent.

Ash 1.25 " $"$ "

Albuminoids 6.26 " "
II. 75.75 per cent. $0.851 "$ " " $5.80 \% "$ " $"$

3.51 per cent. 23.95 " " Ash Or, calculated on the grains after drying :

Albuminoids 24.45 " "

The first were fed to cattle affording the milk analysed under
column I $a$; the second, together with some swill, affording milk II $b$. Eleven cows were fed in the first case in a small stable, very dirty, without bedding. The udders were very dirty, but apparently withont sores.

The cows had been driven into the stalls and secured by causing their heads to pass through a vertical slit, allowing of an up and down motion, and were to be kept their until their udders should dry up. 'Their food appeared to be passing through them with a constant fluid diarrboal discharge. The milk had an alkaline reaction, and a peculiar taste.

In the second case the milk had an exceedingly disagreeable, repulsive taste, 2 slight butyric odor and a strongly alkaline reaction. In fact, the taste gave rise to the impression that soda had been surreptitiously introduced into the milk, without attracting the notice of the inspector who obtained these samples for me. The composition was as follows:

$$
\text { I } t . \quad \text { II } b \text {. }
$$

\begin{tabular}{|c|c|c|}
\hline Fats. & 3.92 & 2.89 \\
\hline 'Total Albuminoids . - & 4.29 & 4.26 \\
\hline (Caseine ............... & 2.74 & $2.32)$ \\
\hline (Albnmen ....... & 1.55 & 1.94) \\
\hline Milk-sugar . . . . . . . & 4.18 & 3.04 \\
\hline 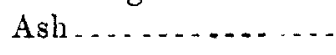 & 0.748 & 0.839 \\
\hline
\end{tabular}


The ratio of the albuminous to the caseinous matters is abnormally high, as if due to the feeding with so large an amount of soluble nitrogenous matter. In the second sample the percentage of fats are abnormally low.

The first milk after 60 hours culture yielded $8 \% 4$ colonies per cubic centimetre; the latter 6500 colonies. After being heated for 15 minutes in the sterilizer, counting from the time when its inside temperature had reached $100^{\circ} \mathrm{C}$, the latter milk had become sterile, yielding no colonies on cuiture.

As to the length of time to which milk should in general be kept at the temperature of boiling water in order to sterilize it, I am in. clined to believe that the period of one hour usually recommended, is excessive. The experiments previously detailen show that as soon as the temperature of milk reached the boiling point, most of the "albumen" is so changed that it becomes precipitable in the cold by dilute acid, and even heating less than half-hour to $100^{\circ}$ C., is sufficient to change the whole of it. 'The remaining albuminoids in milk are similar in their nature to peptones and are not precipitate by dilute acid or by boiling. But the bacteria are made up largely of albuminoids similar to the first two classes of bodies referred to and not to the last, and the probability of their escaping devitalizution after half an hour's boiling is primarily not great. In fact experiments have shown that in the great majority of instances the sterilization is complete in a half hour or less time, and for this reason the prolongation of heating to an hour, with its attendant inconvenienees, js in general an unnecessary precaution.

My thanks are due to Mr. Geo. W. McGuire, the State Dairy Commissioner of New Jersey, and to my assistant, Mr. Wm. G. Johnston, for their aid in the performance of the experiments detailed in this article. 